\title{
Optical coherence tomography in the two-micron wavelength regime for paint and other high opacity material
}

\author{
C. S. Cheung, ${ }^{1}$ J. M. O. Daniel, ${ }^{2,3}$ M. Tokurakawa, ${ }^{2,4}$ W. A. Clarkson, ${ }^{2}$ and H. Liang ${ }^{1 *}$ \\ ${ }^{1}$ School of Science \& Technology, Nottingham Trent University, Nottingham NG11 8NS, UK \\ ${ }^{2}$ Optoelectronics Research Centre, University of Southampton, Highfield, SO17 1BJ, UK \\ ${ }^{3}$ Present address: Aether Photonics, Melbourne, Australia \\ ${ }^{4}$ Present address: Institute for Laser Science, University of Electro-Communications \\ 1-5-1 Chofugaoka, Chofu-shi, Tokyo 182-8585, Japan \\ *Haida.Liang@ntu.ac.uk
}

Received Month X, XXXX; revised Month X, XXXX; accepted Month X,

XXXX; posted Month X, XXXX (Doc. ID XXXXX); published Month X, XXXX

\begin{abstract}
An optical coherence tomography (OCT) system using a compact fiber source emitting amplified spontaneous emission (ASE) at central wavelength of 1960nm with bandwidth of 40nm is developed to enhance the probing depth in highly scattering material with low water content. Examples of application to paint is used to demonstrate significantly improved penetration depth in high opacity material in the two-micron wavelength regime.
\end{abstract}

OCIS Codes: (110.4500) Optical coherence tomography; (120.3180) interferometry; (290.4210) multiple scattering; (140.3070) Infrared and far-infrared lasers; (140.3510) Lasers, fiber http://dx.doi.org/10.1364/OL.99.099999

Optical Coherence Tomography (OCT) is a non-invasive, non-contact imaging technique capable of $3 \mathrm{D}$ imaging of subsurface microstructure using a fast scanning Michelson interferometer [1]. Operating in the near infrared, it was originally designed for in vivo optical biopsy of the eye and other biological tissues [2]. OCT has increasingly found application in the non-biomedical field ranging from industrial applications [3] to art conservation and Archaeology [4]. In the last ten years, OCT has been successfully applied to the non-invasive imaging of historical paintings and other cultural artifacts $[5,6]$. The non-invasive nature of OCT makes it particularly useful for imaging the subsurface structure of cultural artifacts as conservation ethics discourage the taking of samples from objects. In cases where the object is intact, OCT is the only method to probe the crosssectional microstructure without damaging the artifact.

While currently available OCTs have shown potential in this field, they are optimized for biomedical applications. One major limitation of OCT imaging is the probing depth through highly scattering paint. OCT systems for biomedical applications are generally restricted to wavelengths between $800 \mathrm{~nm}$ and $1300 \mathrm{~nm}$ for the best compromise between water absorption and tissue scattering [7]. OCT depth of penetration is limited by the opacity of the material, that is the combined effect of absorption and scattering. For the best probing depth, OCT operational wavelength range should avoid spectral regions with strong absorption and scattering. Multiple scattering will also result in the scattering center being placed deeper in depth than reality, resulting in artifacts that mask the subsurface layer structures. In general, the scattering coefficient of a material decreases with increasing wavelength, hence a long wavelength OCT will have larger probing depth for highly scattering material. Since non-biological material such as paint are highly scattering with very low water content, their transparency are not limited by water absorption as in the case of biological tissues. Therefore, the optimum spectral window for OCT imaging of materials with low water content is likely to be different. A recent survey of the transparency of historical artists' pigments over the spectral range of 400-2400 $\mathrm{nm}$ using reflection spectroscopy has shown that the optimum spectral window for OCT imaging of paint layers is around $2.2 \mu \mathrm{m}$ [8]. Off-the-shelf OCT sources are commonly found around $0.8 \mu \mathrm{m}, 1 \mu \mathrm{m}, 1.3 \mu \mathrm{m}$ and $1.5 \mu \mathrm{m}$ and few OCT systems have been built beyond $1.3 \mu \mathrm{m}$. Nevertheless, there has been some work done in exploring the potential of longer wavelength OCT. Bouma et al. [9] compared the performance of an OCT system imaging a human aorta specimen using a stretched pulse mode-locked Er-doped fiber laser at $1.55 \mu \mathrm{m}$, a Tm-doped silica fiber amplified spontaneous emission source at $1.81 \mu \mathrm{m}$ (between the primary water absorption bands at 1.4-1.50 $\mu \mathrm{m}$ and 1.9 $2.2 \mu \mathrm{m})$ and a superluminescent diode (SLD) at $1.3 \mu \mathrm{m}$ and found that the depth of penetration was similar since the reduced scattering at long wavelength is compensated by the increased water absorption. More recently, OCT imaging at 1.6 and $1.7 \mu \mathrm{m}$ using supercontinuum or swept sources are found to be optimum for providing enhanced depth of penetration for biological material with low water content [10-12].

As a proof of concept, we demonstrate in this paper a time-domain OCT (TDOCT) using a compact broadband Tm-doped fiber amplified spontaneous emission (ASE) 
source (preliminary results were presented at a conference [13]). One of the attractive characteristics of such sources is the increased bandwidth with decreasing power, since low power reduces the chance of damage to the sample which is particularly important in applications to cultural heritage [14].

The ASE source used in these experiments was constructed in-house, providing a compact turn-key source with fiber coupled output. The source was constructed in cladding pumped all-fiber arrangement (Fig. 1) with a $4 \mathrm{~m}$ length of thulium-doped active fiber (1 wt.\% thulium concentration) used as the gain medium. Fiber core and cladding dimensions were 6 and $100 \mu \mathrm{m}$ with numerical apertures of 0.22 and 0.46 respectively. Pump light was provided by a fiber coupled single emitter diode (Lumix) operating at a wavelength of $795 \mathrm{~nm}$. This was coupled into the active fiber through a $2+1$ tapered fiber bundle (TFB). Due to a cladding diameter mismatch between the active fiber and TFB $(125 \mu \mathrm{m}$ into $100 \mu \mathrm{m})$, an estimated $30 \%$ of pump light was lost in this coupling process. Output from the fiber source was taken from the single mode port of the TFB after a fiberized isolator (AdValue). Within this configuration, a flat cleave was used to terminate the active fiber at the end opposite the output end. This choice was made to allow the $4 \%$ back-reflection of any spontaneous emission, increasing the output power and uni-directionality of emitted ASE source [15]. This 4\% feedback, coupled with the feedback suppression of the fiber isolator, was found to be sufficiently low to prevent the onset of parasitic lasing over the range of pump power in use. The imaging system consists of a fiber based timedomain OCT (TDOCT) in the Michelson interferometer configuration (Fig. 2) using a fiber coupler specifically designed for broadband transmission at $2 \mu \mathrm{m}$ manufactured by Gooch \& Housego. The output of the ASE light is coupled into the input arm of the fiber coupler and it is split into the reference and the sample arms. In the sample arm, light is directed onto a X-Y galvo scanning mirror which scans and collects the returning light from the sample using a $50 \mathrm{~mm}$ focusing lens. In the reference arm, the light illuminates a scanning reference mirror. To improve the time of capturing, an 'en face' scanning scheme is employed where the fast scanning is done by the galvo mirror in the transverse direction and the slow scanning in depth is achieved by a motorized linear translation stage (Thorlabs NST150) that the reference mirror is attached to. The Doppler shifted reference light and the sample light are recombined and mixed in the output arm of the fiber coupler and the interference signal is detected by a balanced detector (Thorlabs PDB410, using the Hamamatsu G8372 extended InGaAs detector sensitive from 0.9 to $2.1 \mu \mathrm{m}$ in wavelength). By using a balanced detection scheme, the interferometric signal is enhanced by reducing the intensity noise in the ASE source and the vibration noise [16]. The signal is acquired with a 14 bit digitizer (Spectrum M2i4021) and demodulated to retrieve the fringe envelope.

The ASE source spectrum is shown in Fig. 3a. The central wavelength of the source is $\sim 1960 \mathrm{~nm}$ and the FWHM bandwidth is $\sim 43$ to $38 \mathrm{~nm}$ when operating at a power of 2 to $16 \mathrm{~mW}$. The power incident on the sample is $\sim 1.1 \mathrm{~mW}$ when the source operates at $8 \mathrm{~mW}$ output power. The theoretically expected FWHM axial resolution assuming a Gaussian spectrum is given by $\Delta \mathrm{z} \sim 0.44 \lambda_{0}^{2} / \Delta \lambda$ which is $\sim 40 \mu \mathrm{m}$ in this case. Figure $3 \mathrm{~b}$ shows the interference signal with a mirror in the sample arm demonstrating an axial resolution of $\sim 35 \mu \mathrm{m}$ consistent with the theoretically expected value. The slightly better experimental axial resolution compared with the theoretically expected value can be explained by the simplistic assumption of Gaussian source spectrum without taking into account of the spectral response of the detectors and optical components. It is found that in the 2 $\mu \mathrm{m}$ wavelength range, the requirement of dispersion balancing of the two arms in the Michelson interferometer is not as critical as it is at shorter wavelengths such as $800 \mathrm{~nm}$ and the theoretical axial resolution was achieved without dispersion compensation. A scan of a USAF microscope calibration chart showed the transverse resolution of the OCT to be $\sim 17 \mu \mathrm{m}$. The sensitivity of the system was found to be $\sim-58 \mathrm{~dB}$.

The 1960nm OCT was applied to a highly scattering paint sample of titanium white in linseed oil painted on glass. Figure 4 shows a comparison of the cross-section images obtained with the $1960 \mathrm{~nm}$ OCT (Fig. 4d), a 930 nm OCT (Thorlabs OCP930SR; Fig. 4b) and a $1310 \mathrm{~nm}$ OCT (Zeiss Visante; Fig. 4c) of the sample. The $930 \mathrm{~nm}$ OCT is a Fourier domain OCT (FDOCT) with an axial resolution of $6.5 \mu \mathrm{m}$ and the $1310 \mathrm{~nm}$ OCT is a TDOCT with an axial resolution of $18 \mu \mathrm{m}$. The paint is highly scattering at 930 and $1310 \mathrm{~nm}$, as both the paint/glass interface and the bottom of the glass/air interface are masked by multiply scattered photons. However, it is transparent at $1960 \mathrm{~nm}$ since the paint/glass interface as well as the interface between the bottom of the glass slide and air can be seen in Fig. $4 \mathrm{~d}$.

Some paints have strong absorption in the visible and/or near infrared, for example, Prussian blue absorbs strongly at wavelength less than $1 \mu \mathrm{m}$, copper pigments such as azurite and malachite absorbs strongly at wavelength less than $1.5 \mu \mathrm{m}$ and cobalt pigments have a broad absorption trough at 1.3-1.6 $\mu \mathrm{m}$. Figure 5b and Fig. $5 \mathrm{c}$ shows a comparison of the $930 \mathrm{~nm}$ and $1960 \mathrm{~nm}$ OCT cross-section images for a layer of Prussian blue oil paint over a board prepared with a white gesso ground (Fig. 5a). The Prussian blue paint layer is strongly absorbing at 930 $\mathrm{nm}$ where only the air/paint interface is visible and the paint/ground interface is not visible (Fig. 5b). However, at $1960 \mathrm{~nm}$ the paint layer can be clearly delineated showing some scattering from the paint layer and the paint/ground interface is visible (Fig.5c). Figure 5c shows the Prussian blue layer is $\sim 350 \mu \mathrm{m}$ in optical thickness or $\sim 230 \mu \mathrm{m}$ in physical thickness if we assume the paint refractive index to be $~ 1.5$. Figure 5d shows a zoomed-in color image of a region of the sample where there is a lamp black water color underdrawing beneath the Prussian blue oil paint. The underdrawing cannot be seen beneath the paint in the visible owing to the opacity of Prussian blue. Figure 5e shows a $\sim 2.2 \mu \mathrm{m}$ direct image of the same region obtained with FLIR InSb camera with a bandpass filter (1.7-2.6 $\mu \mathrm{m})$. Figure $5 \mathrm{f}$ shows an average of 'en face' slices extracted from an image cube collected from the same region. The underdrawing is clearly visible in this 
averaged 'en face' slice demonstrating superior contrast and resolution of this OCT en-face image (Fig. 5f) than that obtained from the direct digital imaging at $2.2 \mu \mathrm{m}$ (Fig. 5e). In comparison, a survey of oil paint with OCT imaging at $1.55 \mu \mathrm{m}$ found both titanium white and Prussian blue to be opaque and the paint ground interface was not visible due to the highly scattering and absorbing properties respectively of the paint samples [17].

In conclusion, we have demonstrated a TDOCT in the $2 \mu \mathrm{m}$ regime using an ASE source to have superior probing depth than OCT at shorter wavelength for highly scattering or highly absorbing paint. OCT in the $2 \mu \mathrm{m}$ regime has the potential to increase the probing depth not only for painted objects but also for other highly scattering material with low water content. To improve the speed of acquisition and depth resolution of OCT imaging in the two-micron regime, we are currently developing a Fourier domain OCT using a source with a significantly broader bandwidth.

Funding from UK AHRC and EPSRC Science \& Heritage Programme (Interdisciplinary Research Grant $\mathrm{AH} / \mathrm{H} 032665 / 1$ ) is gratefully acknowledged. We are grateful to the National Gallery for providing paint samples and to Gooch \& Housego plc. for providing the 2 $\mu \mathrm{m}$ fiber couplers.

\section{References}

1. W. Drexler and J. G. Fujimoto, Optical Coherence Tomography: Technology and applications, Springer, Berlin (2008).

2. D. Huang, E. A. Swanson, C. P. Lin, J. S. Schuman, W. G. Stinson, W. Chang, M. R. Hee, T. Flotte, K. Gregory, C. A. Puliafito, and J. G. Fujimoto, Science 254, 1178 (1991).

3. D. Stifter, Appl. Phys. B 88, 337 (2007).

4. H. Liang, B. Peric, M. Hughes, A. Podoleanu, M. Spring, and S. Roehrs, Proc. SPIE 7139, 713915 (2008).

5. H. Liang, M. G. Cid, R. G. Cucu, G. M. Dobre, A. Gh. Podoleanu, J. Pedro and D. Saunders, Opt. Express 13, 6133 (2005).

6. P. Targowski and M. Iwanicka, Appl. Phy. A 106, 265 (2012).

7. A. Alex, B. Povazay, B. Hofer, S. Popov, C. Glittenberg, S. Binder, W. Drexler, J. Biomed. Opt. 15, 026025 (2010)

8. H. Liang, R. Lange, B. Peric, and M. Spring, Appl. Phy. B 111, 589 (2013).

9. B. E. Bouma, L. E. Nelson, G. J. Tearney, D. J. Jones, M. E. Brezinski, and James G. Fujimoto, J. Biomed. Opt. 3, 76 (1998).

10. U. Sharma, E. W. Chang, and S. H. Yun, Opt. Express 16, 19712 (2008).

11. V. M. Kodach, J. Kalkman, D. J. Faber and T. G. Van Leeuwen, Biomed. Opt. Express 1, 176 (2010).

12. S. Ishida and N. Nishizawa, Biomed. Opt. Express 3, 282 (2012).

13. C. S. Cheung, M. Tokurakawa, J. Daniel, W. A. Clarkson, H. Liang, Proc. SPIE 8790, 87900J (2013)

14. D. Shen, L. Pearson, P. Wang, J. Sahu, W. A. Clarkson, Opt. Express 16, 11021 (2008).

15. P. Wang, J. K. Sahu, and W. A. Clarkson, IEEE J. Quantum Electron. 13, 580 (2007).

16. A. Gh. Podoleanu, Appl. Opt. 39, 173 (2000).

17. A. Szkulmowska, M. Góra, M. Targowska, B. Rouba, D. Stifter, E. Breuer, and P. Targowski in Lasers in the
Conservation of Artworks, Springer proceedings in physics 116, 487 (2007).

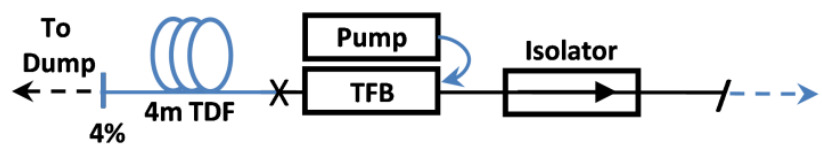

Fig. 1 Cladding pumped all-fiber ASE two-micron source. TDF: Tm3+ doped fiber; TFB: tapered fiber bundle.

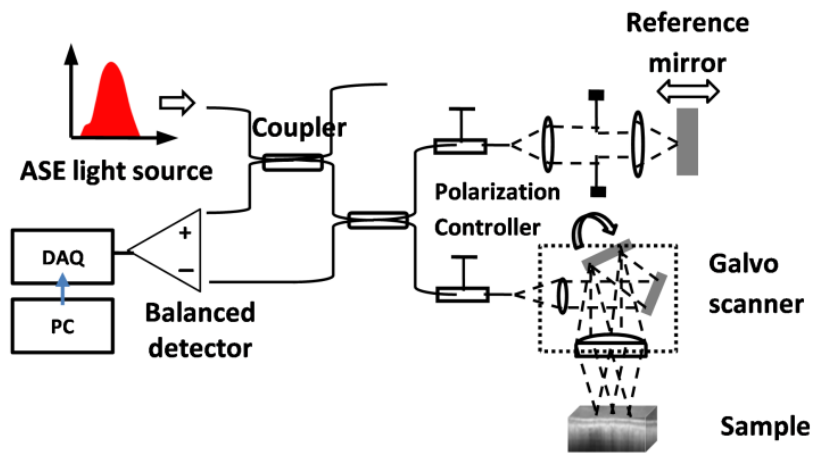

Fig. 2 Schematic diagram of the time domain OCT system.

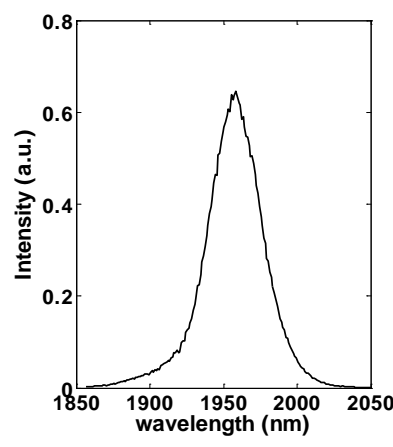

(a)

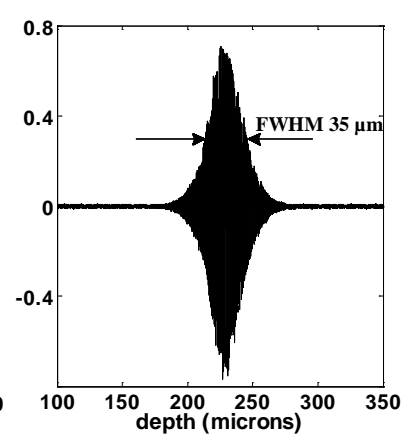

(b)
Fig. 3 a) Spectrum of the Tm doped ASE fiber source; b) Interference fringes with a mirror in the sample arm showing a PSF with FWHM of 35 microns (linear scale). 


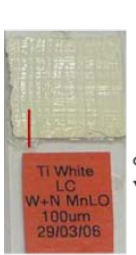

(a)

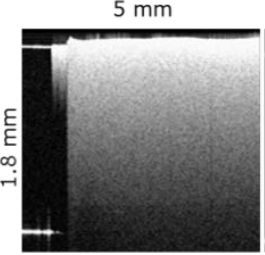

(b)

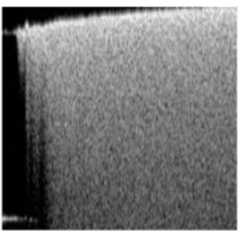

(c)

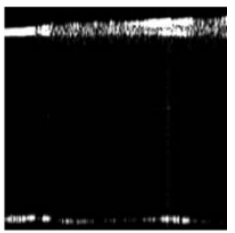

(d)

Fig. 4 a) Titanium white oil paint on a glass microscope slide; b) 930nm Thorlabs FDOCT image showing multiple scattering from Ti White paint; c) 1310nm Zeiss Visante TDOCT; d) 1960nm TDOCT image showing that the paint layer is transparent and even the bottom of the glass slide can be seen.

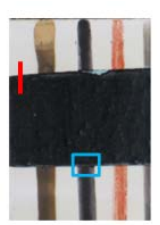

(a)

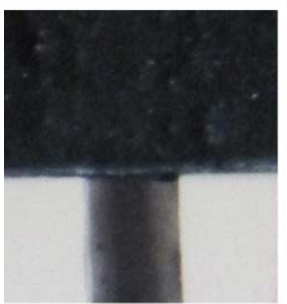

(d)

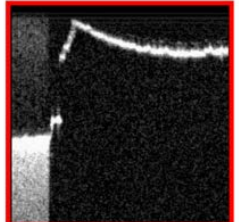

(b)

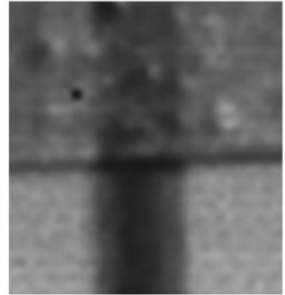

(e)

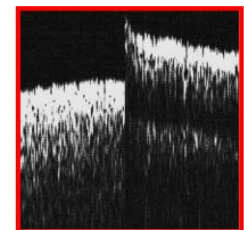

(c)

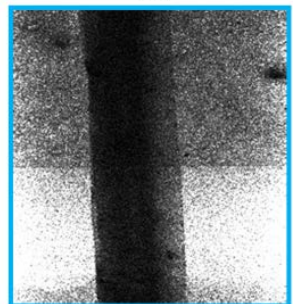

(f)

Fig. 5 a) A panel of white gesso ground with a band of Prussian blue painted over a variety of underdrawing materials; b) a cross-section image produced by a $930 \mathrm{~nm}$ FDOCT at the position marked by the red line segment in a); c) a cross-section image produced by the $1960 \mathrm{~nm}$ TDOCT at roughly the same position as in b); d) Zoomed-in color image of the Prussian blue paint layer over a lamp black water color underdrawings at the position marked by a blue square in a); e) a direct image of the same area as in d) using a FLIR InSb camera with a bandpass filter centered at $2150 \mathrm{~nm}(1700-2600 \mathrm{~nm})$; f) an 'en face' slice of a 1960nm OCT image cube averaged over the layers below the paint to just below the surface of the substrate; Image size in b) and c) are $5 \mathrm{~mm}$ (width) by $0.8 \mathrm{~mm}$ (height) and the image sizes in d), e) and f) are $7 \mathrm{~mm}$ by $7.5 \mathrm{~mm}$. 


\section{Full length references}

1. W. Drexler and J. G. Fujimoto, Optical Coherence Tomograohy: Technology and applications, Springer, Berlin (2008).

2. D. Huang, E. A. Swanson, C. P. Lin, J. S. Schuman, W. G. Stinson, W. Chang, M. R. Hee, T. Flotte, K. Gregory, C. A. Puliafito, and J. G. Fujimoto, "Optical coherence tomography," Science 254, 1178-1181 (1991).

3. D. Stifter, "Beyond biomedicine: a review of alternative applications and developments for optical coherence tomography”, Appl. Phys. B 88, 337 (2007)

4. H. Liang, B. Peric, M. Hughes, A. Podoleanu, M. Spring, and S. Roehrs, "Optical Coherence Tomography in Archaeology and Conservation Science - A new emerging field," Proc. SPIE 7139, 713915 (2008).

5. H. Liang, M. G. Cid, R. G. Cucu, G. M. Dobre, A. Gh. Podoleanu, J. Pedro, and D. Saunders, "Enface Optical Coherence Tomography - a novel application of non-invasive imaging to art conservation", Opt. Express 13, 6133-6144 (2005).

6. P. Targowski and M. Iwanicka, "Optical Coherence Tomography for structural examination of cultural heritage objects and monitoring of restoration processes - a review," Appl. Phy. A 106, 265-277 (2012).

7. A. Alex, B. Povazay, B. Hofer, S. Popov, C. Glittenberg, S. Binder and W. Drexler, "Multispectral in vivo three-dimensional optical coherence tomography of human skin", J. Biomed. Opt. 15, 026025 (2010)

8. H. Liang, R. Lange, B. Peric, and M. Spring "Optimum spectral window for imaging of art with optical coherence tomography," Appl. Phy. B 111, 589-602 (2013).

9. B. E. Bouma, L. E. Nelson, G. J. Tearney, D. J. Jones, M. E. Brezinski, and James G. Fujimoto, "Optical coherence tomography imaging of human tissue at $1.55 \mu \mathrm{m}$ and $1.81 \mu \mathrm{m}$ using Er- and Tm-doped fiber sources”, J. Biomed. Opt. 3, 76-79 (1998).

10. U. Sharma, E. W. Chang, and S. H. Yun, "Longwavelength optical coherence tomography at 1.7 $\mu \mathrm{m}$ for enhanced imaging depth", Opt. Express 16, 19712-19723 (2008).

11. V. M. Kodach, J. Kalkman, D. J. Faber and T. G. Van Leeuwen, "Quantitative comparison of the OCT imaging depth at 1300nm and 1600nm", Biomed. Opt. Express 1, 176 (2010)

12. S. Ishida and N. Nishizawa, "Quantitative comparison of contrast and imaging depth of ultrahigh-resolution optical coherence tomography images in 800-1700 nm wavelength region", Biomed. Opt. Express 3 (2), 282-294 (2012)

13. C. S. Cheung, M. Tokurakawa, J. Daniel, W. A. Clarkson, H. Liang, "Long wavelength optical coherence tomography for painted objects", Proc. SPIE 8790, 87900J (2013)

14. D. Shen, L. Pearson, P. Wang, J. Sahu, W. A. Clarkson, "Broadband Tm-doped superfluorescent fiber source with $11 \mathrm{~W}$ singleended output power," Opt. Express 16, 1102111026 (2008).

15. P. Wang, J. K. Sahu, and W. A. Clarkson, "Power Scaling of Ytterbium-doped Fiber Superfluorescent Sources," IEEE J. Quantum Electron. 13, 580-587 (2007).

16. A. Gh. Podoleanu, "Unbalanced versus balanced operation in an optical coherence tomography system", Applied Optics 39, 173-182 (2000)

17. A. Szkulmowska, M. Góra, M. Targowska, B. Rouba, D. Stifter, E. Breuer, and P. Targowski, "Applicability of optical coherence tomography at $1.55 \mu \mathrm{m}$ to the examination of oil paintings", in Lasers in the Conservation of Artworks, Springer proceedings in physics 116, 487-492 (2007) 